1 Hacettepe Journal of Mathematics and Statistics

$\bigcap$ Volume 45 (5) (2016), 1435-1440

\title{
Compactness and local compactness of the proximal hyperspace
}

\author{
Marco Rosa*†
}

\begin{abstract}
Compactness and local compactness of the hyperspace endowed with both the Vietoris topology and the Hausdorff metric topology, have been characterized by Costantini, Levi and Pelant. Our aim is to characterize these two properties for the proximal topology, which is related to both of the previous topologies.
\end{abstract}

Keywords: Hyperspace, Proximal Topology, Local Compactness, Kuratowski Convergence

2000 AMS Classification: Primary 54B20; Secondary 54D30, 54D45

Received : 14.05.2015 Accepted: 07.01.2016 Doi : 10.15672/HJMS.20164515689

\section{Introduction}

The first one to characterize compactness of the Vietoris topology on the hyperspace $\mathrm{CL}(X)$ of non-empty closed subsets of a topological space $X$, was Michael in [3]. He also gave a result about local compactness, but it was not correct as remarked in the paper [2]. In that paper, Costantini, Levi and Pelant studied compactness and local compactness of several hyperspace topologies. In particular they characterized compactness and local compactness of $\mathrm{CL}(X)$ endowed both with the Vietoris topology $\tau_{V}$ and the Hausdorff metric topology $\tau_{H_{d}}$.

Following the same spirit and using a similar technique, we characterize compactness and local compactness of $\mathrm{CL}(X)$ endowed with the proximal topology $\tau_{\delta(d)}$. We show that both properties are equivalent to compactness of $X$. The choice of $\tau_{\delta(d)}$ is motivated by the fact that it is deeply connected both to $\tau_{V}$ and $\tau_{H_{d}}$, because it can be obtained as supremum of the lower Vietoris topology and the upper Hausdorff metric topology, i.e. $\tau_{\delta(d)}=\tau_{V}^{-} \vee \tau_{H_{d}}^{+}$.

*Dipartimento di Matematica, Informatica ed Economia, Università degli studi della Basilicata, Via dell'Ateneo Lucano 10, 85100 Potenza, Italy. Email: marco.rosa@unibas.it

†Corresponding Author. 


\section{Preliminaries}

Let $X$ be a metrizable space. Given a metric $d$ on $X$, we denote by $D_{d}$ the gap between two non-empty closed sets $E, F \in \mathrm{CL}(X)$, defined as:

$$
D_{d}(E, F)=\inf _{x \in E} \inf _{y \in F} d(x, y) .
$$

Let $x \in X$, we denote by $B_{\varepsilon}(x)$ the open ball of radius $\varepsilon$ and center $x$. Given $A \in \operatorname{CL}(X)$, we denote by $B_{\varepsilon}[A]$ the $\varepsilon$-expansion of $A$, i.e. $B_{\varepsilon}[A]=\bigcup_{a \in A} B_{\varepsilon}(a)$. It is easy to check that $B_{\frac{\varepsilon}{2}}\left[B_{\frac{\varepsilon}{2}}[A]\right] \subseteq B_{\varepsilon}[A]$ and $B_{\frac{\varepsilon}{3}}\left[\overline{B_{\frac{\varepsilon}{3}}}[A]\right] \subseteq B_{\varepsilon}[A]$ for every $\varepsilon>0$ and every $A \in \mathrm{CL}(X)$.

Recall that the Vietoris topology is $\tau_{V}=\tau_{V}^{-} \vee \tau_{V}^{+}$, where $\tau_{V}^{-}$and $\tau_{V}^{+}$are generated respectively by the collection of all $V^{-}=\{F \in \mathrm{CL}(X) \mid F \cap V \neq \varnothing\}$ and $W^{+}=\{F \in$ $\mathrm{CL}(X) \mid F \subseteq W\}$, when $V$ and $W$ run over all the open subsets of $X$. The Hausdorff metric topology $\tau_{H_{d}}$ is generated by the Hausdorff distance on $\mathrm{CL}(X)$ induced by $d$ (see for instance [1]). A base for $\tau_{H_{d}}^{+}$is constituted by the collection of all $W^{++}=\{F \in$ $\left.\mathrm{CL}(X) \mid D_{d}(F, X \backslash W)>0\right\}=\left\{F \in \mathrm{CL}(X) \mid \exists \varepsilon>0: B_{\varepsilon}[F] \subseteq W\right\}$, when $W$ runs through the open subsets of $X$. As recalled before, the proximal topology $\tau_{\delta(d)}$ is the supremum of the lower Vietoris topology and the upper Hausdorff metric topology, i.e. $\tau_{\delta(d)}=\tau_{V}^{-} \vee \tau_{H_{d}}^{+}$.

Recall that the a net $\left(C_{i}\right)_{i \in I}$ is convergent to $C$ with respect to the Kuratowski convergence if, and only if, it converges with respect to $\tau_{V}^{-}$and $C \supseteq \operatorname{Ls}_{i \in I} C_{i}$ where, denoted by $\mathfrak{U}(x)$ the collection of open neighbourhoods of $x$,

$$
\operatorname{Ls}_{i \in I} C_{i}=\left\{x \in X \mid \forall V \in \mathfrak{U}(x) \forall i \in I \exists j_{i} \succeq i: V \cap C_{j_{i}} \neq \varnothing\right\}=\bigcap_{i \in I} \overline{\bigcup_{j \succeq i} C_{j}} .
$$

Given a metrizable space $X$, we denote by $\mathfrak{M}(X)$ the set of all compatible metrics on $X$.

\section{The main result}

In the sequel, in the definition of compactness and local compactness we require the space also to be Hausdorff.

Our main result is the following theorem.

3.1. Theorem. Let $X$ be a metrizable space, let $C \in \mathrm{CL}(X)$ and $d \in \mathfrak{M}(X)$. Then $\mathrm{CL}(X)$ is $\tau_{\delta(d)}$-locally compact at $C$ if, and only if, there exists $\varepsilon>0$ such that $\overline{B_{\varepsilon}[C]}$ is compact.

As a consequence we can characterize both local compactness and compactness of $X$, using conditions on $\mathrm{CL}(X)$. Moreover we can also characterize compactness of $\left(\mathrm{CL}(X), \tau_{\delta(d)}\right)$, showing that it is equivalent to local compactness of $\left(\mathrm{CL}(X), \tau_{\delta(d)}\right)$.

3.2. Corollary. Let $X$ be a metrizable space. $X$ is locally compact if, and only if, CL $(X)$ is $\tau_{\delta(d)}$-locally compact at $\{x\}$, for every $x \in X$.

3.3. Corollary. Let $X$ be a metrizable space. The following are equivalent:

(1) $X$ is compact;

(2) $\left(\mathrm{CL}(X), \tau_{\delta(d)}\right)$ is locally compact;

(3) $\left(\mathrm{CL}(X), \tau_{\delta(d)}\right)$ is locally compact at $X$.

Proof. The implications $(1) \Rightarrow(2)$ and $(3) \Rightarrow(1)$ follow from Theorem 3.1, while $(2) \Rightarrow$ (3) is obvious.

The following result was proved in [2, Theorem 11]. 
3.4. Theorem. Let $X$ be a regular space and let $C \in \mathrm{CL}(X)$. Then $\mathrm{CL}(X)$ is $\tau_{V}$-locally compact at $C$ if, and only if, there exists an open set $A \subseteq X$ such that $C \subseteq A$ and $\bar{A}$ is compact.

Combining the previous result and our Theorem 3.1, we obtain as a consequence the equivalence of local compactness and compactness of proximal and Vietoris topologies.

3.5. Corollary. Let $X$ be a metrizable space. The following are equivalent:

(1) $X$ is compact;

(2) $\left(\mathrm{CL}(X), \tau_{\delta(d)}\right)$ is compact;

(3) $\left(\mathrm{CL}(X), \tau_{V}\right)$ is compact;

(4) $\left(\mathrm{CL}(X), \tau_{\delta(d)}\right)$ is locally compact;

(5) $\left(\mathrm{CL}(X), \tau_{V}\right)$ is locally compact;

Proof. The equivalence between $(1) \Leftrightarrow(3) \Leftrightarrow(5)$ has been proved in [2, Corollary 13]. If $X$ is compact, then $\left(\mathrm{CL}(X), \tau_{\delta(d)}\right)$ is compact since $\tau_{\delta(d)}=\tau_{V}$ and this proves the implication $(1) \Rightarrow(2)$. The implication $(2) \Rightarrow(4)$ is obvious. Finally the equivalence $(1) \Leftrightarrow(4)$ follows from Corollary 3.3.

3.6. Remark. Note that $(4) \Rightarrow(5)$ can be easily proved in a direct way, in order to explicitly use the condition that characterize local compactness of $\tau_{\delta(d)}$ and $\tau_{V}$. Indeed, if $\left(\mathrm{CL}(X), \tau_{\delta(d)}\right)$ is locally compact at $C$, then by Theorem 3.1 there exists $\varepsilon>0$ such that $\overline{B_{\varepsilon}[C]}$ is compact. Then $V=B_{\varepsilon}[C]$ is an open set containing $C$ and with compact closure. Hence $\left(\mathrm{CL}(X), \tau_{V}\right)$ is locally compact at $C$ by Theorem 3.4.

In [2] it has been proved that local compactness of the Vietoris hyperspace is in general a strictly stronger condition than local compactness of the Hausdorff hyperspace. As a consequence of Corollary 3.5, local compactness of the proximal hyperspace has the same behaviour. Finally it has been proved in [1, Theorem 3.2.4] that compactness of the Hausdorff hyperspace is equivalent to compactness of $X$, and by Corollary 3.5, this is equivalent to compactness of the proximal hyperspace.

\section{Proof our main result}

To prove our main theorem we need several preliminary results. The following remarks are of easy verification.

4.1. Remark. Let $X$ be a metrizable space and let $d \in \mathfrak{M}(X)$. If $C$ is closed, then $C^{+}$ is $\tau_{\delta(d)}$-closed. If $K$ is compact, then $(X \backslash K)^{++}=(X \backslash K)^{+}$and therefore $(X \backslash K)^{+}$ is $\tau_{\delta(d)}$-open and $K^{-}$is $\tau_{\delta(d)}$-closed.

Proof. Since $X \backslash C$ is open, $\mathrm{CL}(X) \backslash C^{+}=(X \backslash C)^{-}$is $\tau_{\delta(d)}$-open.

If $K$ is compact and $F$ is closed, then $F \cap K=\varnothing$ implies $D_{d}(F, K)>0$. Therefore $(X \backslash K)^{+}=\{F \mid F \subseteq X \backslash K\}=\left\{F \mid D_{d}(F, K)>0\right\}=(X \backslash K)^{++}$. Moreover $\mathrm{CL}(X) \backslash K^{-}=(X \backslash K)^{+}=(X \backslash K)^{++}$which is $\tau_{\delta(d)}$-open.

4.2. Remark. Let $X$ be a metrizable space and let $d \in \mathfrak{M}(X)$. Then $\left(\operatorname{CL}(X), \tau_{\delta(d)}\right)$ is $\mathrm{T}_{2}$.

Proof. Let $A, C \in \mathrm{CL}(X)$ such that $A \neq C$. We may suppose there exists $a \in A \backslash C$. Since $a \notin \bigcap_{\varepsilon>0} \overline{B_{\varepsilon}[C]}=C$, there exists $\varepsilon>0$ such that $a \in X \backslash \overline{B_{\varepsilon}[C]}$. Take $\delta>0$ such that $B_{\delta}(a) \subseteq X \backslash \overline{B_{\varepsilon}[C]}$. We claim that $B_{\varepsilon}[C] \subseteq X \backslash \overline{B_{\frac{\delta}{2}}(a)}$. Indeed let on the contrary $x \in B_{\varepsilon}[C] \cap \overline{B_{\frac{\delta}{2}}(a)}$. There exists $y \in B_{\frac{\delta}{2}}(x) \cap B_{\frac{\delta}{2}}(a)$, hence $d(x, a) \leq d(x, y)+d(y, a)<\delta$. Then $x \in B_{\delta}(a) \subseteq X \backslash \overline{B_{\varepsilon}[C]}$, impossible. 
Hence $B_{\varepsilon}[C] \subseteq X \backslash \overline{B_{\frac{\delta}{2}}(a)}$, that is $C \in\left(X \backslash \overline{B_{\frac{\delta}{2}}(a)}\right)^{++}$which is $\tau_{\delta(d)}$-open. Moreover $A \in B_{\frac{\delta}{2}}(a)^{-}$which is $\tau_{\delta(d)^{-}}$open and $B_{\frac{\delta}{2}}(a)^{-} \cap\left(X \backslash \overline{B_{\frac{\delta}{2}}(a)}\right)^{++} \subseteq B_{\frac{\delta}{2}}(a)^{-} \cap(X \backslash$ $\left.\overline{B_{\frac{\delta}{2}}(a)}\right)^{+} \subseteq B_{\frac{\delta}{2}}(a)^{-} \cap\left(X \backslash B_{\frac{\delta}{2}}(a)\right)^{+}=\varnothing$.

The following result gives a sufficient condition for compactness of a collection $\mathcal{K} \subseteq$ $\mathrm{CL}(X)$.

4.3. Proposition. Let $X$ be a metrizable space. Let $\mathcal{K} \subseteq \mathrm{CL}(X)$ and $d \in \mathfrak{M}(X)$. If $\mathcal{K}$ is $\tau_{\delta(d)}$-closed, and for every $F \in \mathrm{CL}(X)$, for every $\varepsilon>0$ and for every open cover $\mathcal{U}$ of $\overline{B_{\varepsilon}[F]}$, there exists a finite open subcover $\mathcal{F}$ such that

$$
\mathcal{K} \cap \overline{B_{\varepsilon}[F]}-\subseteq \bigcup_{U \in \mathcal{F}} U^{-},
$$

then $\mathcal{K}$ is $\tau_{\delta(d)}$-compact.

Proof. Let $\left(C_{j}\right)_{j \in J}$ be a net in $\mathcal{K}$. By Remark 4.2 , we have to prove that it has a convergent subnet. By [1, Theorem 5.2.11], there exists a subnet $\left(C_{j_{i}}\right)_{i \in I}$ which is $K$ convergent to a set $C \in \mathrm{CL}(X) \cup\{\varnothing\}$. Note that since $\{\varnothing\}=\varnothing^{++}, \varnothing$ is isolated with respect to $\tau_{\delta(d)}$ and therefore $C \in \mathrm{CL}(X)$. Moreover $\left(C_{j_{i}}\right)_{i \in I}$ is $\tau_{V}^{-}$-convergent to $C$. We want to prove that $C_{j_{i}} \rightarrow C$ with respect to $\tau_{H_{d}}^{+}$, and this would also imply that $C \in \mathcal{K}$ since $\mathcal{K}$ is $\tau_{\delta(d)}$-closed.

On the contrary, suppose there exists $W$ open such that $C \in W^{++}$but $C_{j_{i}} \notin W^{++}$ frequently. Since $D_{d}(C, X \backslash W)>0$, there exists $\varepsilon>0$ such that $\overline{B_{\varepsilon}[X \backslash W]} \subseteq X \backslash C$. Since $C \supseteq \operatorname{Ls}_{i \in I} C_{j_{i}}$, for every $x \in \overline{B_{\varepsilon}[X \backslash W]} \subseteq X \backslash C \subseteq X \backslash \operatorname{Ls}_{i \in I} C_{j_{i}}$, there exist a neighbourhood $V_{x}$ of $x$, and a index $i_{x} \in I$ such that $V_{x} \cap C_{j_{i}}=\varnothing$ for every $i \geq i_{x}$. Since $\overline{B_{\varepsilon}[X \backslash W]} \subseteq \bigcup_{x \in \overline{B_{\varepsilon}[X \backslash W]}} V_{x}$, by hypothesis there exist $x_{1}, \ldots, x_{n} \in \overline{B_{\varepsilon}[X \backslash W]}$ such that

$$
\mathcal{K} \cap \overline{B_{\varepsilon}[X \backslash W]} \subseteq \bigcup_{k=1}^{n} V_{x_{k}}^{-} .
$$

Let $i_{0} \in I$ such that $i_{0} \geq i_{x_{k}}$ for $k=1, \ldots, n$. Then for every $i \geq i_{0}, C_{j_{i}} \cap V_{x_{k}}=\varnothing$ for every $k=1, \ldots, n$, that is $C_{j_{i}} \notin \bigcup_{k=1}^{n} V_{x_{k}}^{-}$. Since $C_{j_{i}} \in \mathcal{K}$, then $C_{j_{i}} \notin \overline{B_{\varepsilon}[X \backslash W]}$ for every $i \geq i_{0}$, that is $C_{j_{i}} \cap \overline{B_{\varepsilon}[X \backslash W]}=\varnothing$.

On the other hand, $C_{j_{i}} \notin W^{++}$frequently, so that there exists $k \geq i_{0}$, such that for every $\delta>0, B_{\delta}\left[C_{j_{k}}\right] \cap(X \backslash W) \neq \varnothing$. In particular for $\delta=\varepsilon$, there exist $y \in C_{j_{k}}$, $z \in X \backslash W$ such that $d(z, y)<\varepsilon$. But then $y \in C_{j_{k}} \cap B_{\varepsilon}[X \backslash W] \neq \varnothing$, a contradiction.

4.4. Lemma. Let $X$ be a metrizable space. Let $K \in \mathrm{CL}(X)$ and $d \in \mathfrak{M}(X)$. If $K$ is compact and there exists $\delta>0$ such that $\overline{B_{\delta}(x)}$ is compact for every $x \in K$, then $\overline{B_{\frac{\delta}{2}}[K]}$ is compact.

Proof. Of course $K \subseteq \bigcup_{x \in K} B_{\frac{\delta}{2}}(x)$. Since $K$ is compact there exist $x_{1}, \ldots, x_{n} \in K$ such that $K \subseteq \bigcup_{i=1}^{n} B_{\frac{\delta}{2}}\left(x_{i}\right)$. Then $B_{\frac{\delta}{2}}[K] \subseteq \bigcup_{i=1}^{n} B_{\delta}\left(x_{i}\right)$ and therefore $\overline{B_{\frac{\delta}{2}}[K]} \subseteq$ $\overline{\bigcup_{i=1}^{n} B_{\delta}\left(x_{i}\right)}=\bigcup_{i=1}^{n} \overline{B_{\delta}\left(x_{i}\right)}$ which is compact. Hence $\overline{B_{\frac{\delta}{2}}[K]}$ is compact.

4.5. Lemma. Let $X$ be a metrizable space. Let $K \in \mathrm{CL}(X)$ and $d \in \mathfrak{M}(X)$. If $K$ is compact and there exists $\delta>0$ such that $\overline{B_{\delta}(x)}$ is compact for every $x \in K$, then ${\overline{B_{\frac{\delta}{2}}[K]}}^{+}$ is $\tau_{\delta(d)}$-compact.

Proof. The set ${\overline{B_{\frac{\delta}{2}}[K]}}^{+}$is $\tau_{\delta(d)}$-closed by Remark 4.1. Let $C \in \mathrm{CL}(X), \varepsilon>0$ and let $\mathcal{U}$ be an open cover of $\overline{B_{\varepsilon}[C]}$. By Proposition 4.3, we have to find a finite open subcover $\mathcal{F}$ 
such that ${\overline{B_{\frac{\delta}{2}}[K]}}^{+} \cap{\overline{B_{\varepsilon}[C]}}^{-} \subseteq \bigcup_{U \in \mathcal{F}} U^{-}$. If $\overline{B_{\varepsilon}[C]} \cap \overline{B_{\frac{\delta}{2}}[K]}=\varnothing$ then ${\overline{B_{\varepsilon}[C]}}^{-} \cap{\overline{B_{\frac{\delta}{2}}[K]}}^{+}$ is empty. Suppose $\overline{B_{\varepsilon}[C]} \cap \overline{B_{\frac{\delta}{2}}[K]} \neq \varnothing$. Note that $\overline{B_{\varepsilon}[C]} \cap \overline{B_{\frac{\delta}{2}}[K]}$ is compact since it is closed and it is contained in $\overline{B_{\frac{\delta}{2}}[K]}$ which is compact by Lemma 4.4. There exist $U_{1}, \ldots, U_{n} \in \mathcal{U}$ such that $\overline{B_{\varepsilon}[C]} \cap \overline{B_{\frac{\delta}{2}}[K]} \subseteq \bigcup_{i=1}^{n} U_{i}$. Therefore ${\overline{B_{\varepsilon}[C]}}^{-} \cap{\overline{B_{\frac{\delta}{2}}[K]}}^{+} \subseteq$ $\left(\overline{B_{\varepsilon}[C]} \cap \overline{B_{\frac{\delta}{2}}[K]}\right)^{-} \subseteq \bigcup_{i=1}^{n} U_{i}^{-}$.

4.6. Proposition. Let $X$ be a metrizable space and $d \in \mathfrak{M}(X)$. Let $V, V_{1}, \ldots V_{k}$ be any non-empty set. If $\mathcal{V}=\bigcap_{i=1}^{k} V_{i}^{-} \cap V^{+}$is $\tau_{\delta(d)}$-compact and non-empty, then $V$ is compact.

Proof. Since $\mathcal{V} \neq \varnothing$, for every $i=1, \ldots, k$ we can fix $x_{i} \in V_{i} \cap V$.

We first prove that $V$ is closed. Otherwise there should exist $\left(y_{n}\right)_{n \in \mathbb{N}}$ such that $y_{n} \in V$ and $y_{n} \rightarrow y \in X \backslash V$. Since $\mathcal{V} \neq \varnothing$, for every $i=1, \ldots, k$ we can find $x_{i} \in V_{i} \cap V$. For every $n \in \mathbb{N}$ set $C_{n}=\left\{x_{1}, \ldots, x_{k}, y_{n}\right\}$ and $C=\left\{x_{1}, \ldots, x_{k}, y\right\}$. Note that $C \notin \mathcal{V}$ because $y \in C \backslash V$. Moreover $C_{n} \in \mathcal{V}$ for every $n \in \mathbb{N}$. We prove that $C_{n} \rightarrow_{\tau_{\delta(d)}} C$ in order to have a contradiction, since $\mathcal{V}$ is $\tau_{\delta(d)}$-compact and hence closed.

Let $\mathcal{U}=\bigcap_{i=1}^{p} U_{i}^{-} \cap U^{++}$be a $\tau_{\delta(d)}$-neighbourhood of $C$. Let $i \in\{1, \ldots, p\}$; we distinguish two cases. If there exists $j \in\{1, \ldots, k\}$ such that $x_{j} \in U_{i}$, then $x_{j} \in C_{n} \cap U_{i}$, hence $C_{n} \in U_{i}^{-}$for every $n \in \mathbb{N}$. If $x_{j} \notin U_{i}$ for every $j=1, \ldots, k$, since $C \cap U_{i} \neq 0$, then $y \in C \cap U_{i}$. Take $\varepsilon>0$ such that $B_{\varepsilon}(y) \in U_{i}$. Then $y_{n} \in B_{\varepsilon}(y)$ eventually and therefore $C_{n} \in U_{i}^{-}$eventually. Since $C \in U^{++}$, there exists $\delta>0$ such that $B_{\delta}[C] \subseteq U$. Eventually $y_{n} \in B_{\frac{\delta}{2}}(y)$ and therefore $B_{\frac{\delta}{2}}\left[C_{n}\right] \subseteq B_{\delta}[C] \subseteq U$, that is $C_{n} \in U^{++}$. Hence $C_{n} \in \bigcap_{i=1}^{p} U_{i}^{-} \cap U^{++}$eventually, that is $C_{n} \rightarrow_{\tau_{\delta(d)}} C$.

We now prove that $V$ is compact. Otherwise, there should exist $\left(a_{n}\right)_{n \in \mathbb{N}}$ in $V$ with no cluster point. That is for every $x \in X$ there exists a neighbourhood $V_{x}$ of $x$ and $\nu_{x} \in \mathbb{N}$ such that for every $n \geq \nu_{x}, a_{n} \notin V_{x}$. For every $n \in \mathbb{N}$ set $C_{n}=\left\{x_{1}, \ldots, x_{k}, a_{n}\right\}$. We will prove that $\left(C_{n}\right)_{n \in \mathbb{N}}$ has no cluster point in order to have a contradiction since $\mathcal{V}$ is compact. Let $C \in \mathrm{CL}(X)$.

- If $C \subseteq\left\{x_{1}, \ldots, x_{k}\right\}$, then $\left(\bigcup_{i=1}^{k} V_{x_{i}}\right)^{++}$is a $\tau_{\delta(d)}$-neighbourhood of $C$. On the other hand if $\nu \geq \max \left\{\nu_{x_{1}}, \ldots, \nu_{x_{k}}\right\}$, then for every $n \geq \nu, a_{n} \notin V_{x_{i}}$ for every $i=1, \ldots, k$. Then $C_{n} \notin\left(\bigcup_{i=1}^{k} V_{x_{i}}\right)^{++}$eventually.

- If there exists $x_{0} \in C \backslash\left\{x_{1}, \ldots, x_{k}\right\}$, let $W$ be a neighbourhood of $x_{0}$ such that $W \cap\left\{x_{1}, \ldots, x_{k}\right\}=\varnothing$. Then $C_{n} \notin\left(W \cap V_{x_{0}}\right)^{-}$for every $n \geq \nu_{x_{0}}$, while $C \in\left(W \cap V_{x_{0}}\right)^{-}$.

We are now able to finally prove our main result.

Proof of Theorem 3.1.

$\Rightarrow)$ Suppose that $\left(\mathrm{CL}(X), \tau_{\delta(d)}\right)$ is locally compact at $C$. There exists a neighbourhood $\mathcal{V}=\bigcap_{i=1}^{n} V_{i}^{-} \cap V^{++}$of $C$ such that $\overline{\mathcal{V}}$ is $\tau_{\delta(d)}$-compact. For every $i=1, \ldots, n$, let $x_{i} \in V_{i} \cap C$ and let $\delta>0$ such that $B_{\delta}[C] \subseteq V$. Set $\mathcal{U}=\bigcap_{i=1}^{n}\left\{x_{i}\right\}^{-} \cap{\overline{B_{\frac{\delta}{3}}}[C]^{+}}^{+}$. Note that $\mathcal{U}$ is $\tau_{\delta(d)^{-}}$-closed by Remark 4.1. If $T \in \mathcal{U}$, then $B_{\frac{\delta}{3}}[T] \subseteq B_{\frac{\delta}{3}}\left(\frac{3}{B_{\frac{\delta}{3}}}[C]\right) \subseteq B_{\delta}[C] \subseteq V$, and this implies $\mathcal{U} \subseteq \mathcal{V}$. Hence $\mathcal{U}$ is $\tau_{\delta(d)}$-compact being $\tau_{\delta(d)}$-closed and contained in the $\tau_{\delta(d)}$-compact set $\overline{\mathcal{V}}$. By Proposition $4.6, \overline{B_{\frac{\delta}{3}}[C]}$ is compact.

$\Leftrightarrow)$ Since $C$ is closed and contained in the compact set $\overline{B_{\varepsilon}[C]}$, it is compact. Moreover for every $x \in C, \overline{B_{\varepsilon}(x)}$ is compact since it is contained in $\overline{B_{\varepsilon}[C]}$. By Lemma $4.5,{\overline{B_{\frac{\varepsilon}{2}}}[C]}^{+}$is $\tau_{\delta(d)}$-compact. Moreover $C \in B_{\frac{\varepsilon}{2}}[C]^{++} \subseteq{\overline{B_{\frac{\varepsilon}{2}}}[C]}^{+}$. The 
set $B_{\frac{\varepsilon}{2}}[C]^{++}$is a $\tau_{\delta(d)}$-neighbourhood of $C$ and its closure in $\mathrm{CL}(X)$ is compact since it is contained in ${\overline{B_{\frac{\varepsilon}{2}}[C]}}^{+}$, which is $\tau_{\delta(d)}$-compact. Hence $\left(\mathrm{CL}(X), \tau_{\delta(d)}\right)$ is locally compact at $C$.

\section{References}

[1] Beer, G.: Topologies on closed and closed convex sets, Mathematics and its Applications, No. 268, Kluwer Academic Publishers Group, Dordrecht, 1993.

[2] Constantini, C.-Levi, S.-Pelant, J.: Compactness and local compactness in hyperspaces, Topology Appl. 123(3) (2002), 573-608.

[3] Michael, E.: Topologies on spaces of subsets, Trans. Amer. Math. Soc. 71 (1951), 152-182. 